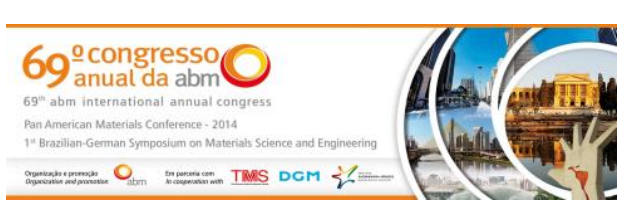

Tema: Engenharia de superfície

\title{
ESTUDO DE PARÂMETROS DO PROCESSO DE OBTENÇÃO DE REVESTIMENTOS DE CONVERSÃO NANOCERÂMICOS À BASE DE TITÂNIO E ZIRCÔNIO*
}

Maíra de Paula Pereira de Lucena ${ }^{1}$ Jane Zoppas Ferreira²

\section{Resumo}

Revestimentos de conversão nanocerâmicos são obtidos pela imersão de peças metálicas em soluções contendo íons de titânio, zircônio, cério entre outros. Esses revestimentos vêm sendo estudados como uma alternativa a outros processos de conversão que possuem inconvenientes ambientais, como a cromatização e a fosfatização. No presente trabalho foram formuladas diferentes soluções a base dos ácidos hexafluorozircônio e hexafluortitânio para a deposição de revestimentos nanocerâmicos sobre substratos de aço galvanizado. O objetivo é mapear os efeitos dos parâmetros de processo de obtenção do revestimento quanto ao desempenho contra corrosão. Os parâmetros estudados foram a concentração, o pH e o tempo de imersão nas soluções formuladas. Para avaliar os revestimentos obtidos foram realizados ensaios eletroquímicos de espectroscopia de impedância complexa. Os resultados indicam uma forte interação entre $0 \mathrm{pH}$ e a concentração dos ácidos em solução e que concentrações mais baixas geram uma proteção mais eficaz $\mathrm{O}$ melhor conjunto de parâmetros foi para menor $\mathrm{pH}$, concentração e tempo de imersão.

Palavras-chave: Revestimentos nanocerâmicos; Conversão; Corrosão.

\section{STUDY OF PROCESS PARAMETERS FOR OBTATION OF NANO-CERAMIC CONVERSION COATINGS BASED ON TITANIUM AND ZIRCONIUM}

\section{Abstract}

Nano-ceramic conversion coatings are obtained by dipping metal cupons in solutions containing ions such as titanium, zirconium, cerium and others. This kind of coating have been studied as an alternative to conventional conversion coatings that presents environmental risks such as chromatization and phosphatization. In the present work galvanized steel substrates were dipped in hexafluorozirconic and hexafluortitanic acids based solutions to produce nano-ceramic conversion coatings. The aim of this work is to map the effects of process parameters on the coating corrosion performance. The parameters studied were the concentration, $\mathrm{pH}$ and immersion time in the solutions formulated. To evaluate the coatings obtained electrochemical impedance spectroscopy curves were performed. The results indicate a strong interaction between $\mathrm{pH}$ and the concentration of acid in solution and that lower concentrations generate more effective protection. The best set of parameters was lower $\mathrm{pH}$, lower concentration and lower immersion time.

Keywords: Nano-ceramic coatings; Conversion coatings; Corrosion.

1 Engenheira de Materiais, Mestranda, Laboratório de Corrosão, Programa de Pós-Graduação em Engenharia de Minas, Materiais e Metalurgia, Universidade Federal do Rio Grande do Sul, Porto Alegre, RS, Brasil.

2 Química, Doutora, Professora, Laboratório de Corrosão, Programa de Pós-Graduação em Engenharia de Minas, Materiais e Metalurgia, Universidade Federal do Rio Grande do Sul, Porto Alegre, RS, Brasil.

* Contribuição técnica ao 69ำ Congresso Anual da ABM - Internacional e ao 14ํㅡㄹ ENEMET - Encontro Nacional de Estudantes de Engenharia Metalúrgica, de Materiais e de Minas, 21 a 25 de julho de 2014, São Paulo, SP, Brasil. 


\section{INTRODUÇÃO}

Os revestimentos de conversão nanocerâmicos vêm sendo estudados como uma alternativa a outros processos de conversão que possuem inconvenientes ambientais, como a cromatização e a fosfatização [1]. Esses revestimentos são obtidos pela imersão de peças metálicas em soluções contendo íons de titânio, zircônio, cério entre outros. São considerados revestimentos de conversão, pois reagem com o substrato: os átomos da superfície são atacados pela solução de conversão; sobre as regiões onde ocorrem as reações catódicas há um aumento local do $\mathrm{pH}$, que causa a precipitação dos íons de interesse presentes na solução na forma de óxidos e hidróxidos. Esses revestimentos têm sido chamados de revestimentos nanocerâmicos [2], devido a sua natureza inorgânica e espessura, na ordem de dezenas de nanômetros. Os nanocerâmicos vêm sendo desenvolvidos para ligas de alumínio [35], magnésio [6], aços [2] e aços galvanizados [7,8].

$\mathrm{O}$ pH do banho de conversão é um dos fatores que interferem na deposição do filme. Ele que controla a espessura da interface de difusão alcalina formada adjacentemente às áreas catódicas. Quando o pH é muito baixo, a deposição do filme não é efetiva, pois a interface de difusão alcalina é menor [9]. Porém o pH deve ser mantido abaixo do valor limite em que ocorre a precipitação completa dos íons de interesse em solução.

A concentração é outro fator importante para a obtenção de um revestimento de qualidade. As soluções mais eficazes em geral são as menos agressivas ao substrato. Isso porque as soluções para conversão de revestimentos nanocerâmicos geralmente contém o ânion fluoreto, que tem um efeito negativo na formação do filme. Em altas concentrações deste ânion o filme desaparece progressivamente [4,9].

O tempo de imersão do substrato influencia a qualidade final da camada depositada: em tempos curtos, em geral menores que $60 \mathrm{~s}$, a camada é heterogênea e apresenta muitas falhas. Após $90 \mathrm{~s}$ o recobrimento total ocorre e inicia-se o crescimento da camada. Porém para tempos acima de $300 \mathrm{~s}$, a camada se torna espessa, porosa e pouco aderente, rompendo e não protegendo mais contra corrosão [3,9].

Além dos três fatores citados, a temperatura e a agitação da solução são considerados parâmetros que influenciam a qualidade e capacidade de proteção do revestimento obtido $[3,5,10]$.

No presente trabalho foram formuladas diferentes soluções a base dos ácidos hexafluorozircônio $\left(\mathrm{H}_{2} \mathrm{ZrF}_{6}\right)$ e hexafluortitânio $\left(\mathrm{H}_{2} \mathrm{TiF}_{6}\right)$ para a deposição de revestimentos nanocerâmicos sobre substratos de aço galvanizado seguindo um planejamento de experimentos tipo fatorial $2 \mathrm{k}$. O processo experimental desta metodologia consiste em realizar ensaios com cada uma das combinações da matriz experimental, para, em seguida, determinar e interpretar os efeitos principais e de interação dos fatores investigados e assim, poder identificar as condições experimentais que interferem no desempenho do produto ou processo [11]. O objetivo é mapear os efeitos dos parâmetros de processo de obtenção do revestimento quanto ao desempenho contra corrosão. Os parâmetros estudados foram as concentrações dos ácidos, o pH e o tempo de imersão nas soluções formuladas. Para avaliar os revestimentos obtidos foram realizados ensaios eletroquímicos de espectroscopia de impedância complexa.

\section{MATERIAIS E MÉTODOS}

\footnotetext{
* Contribuição técnica ao 69ำ Congresso Anual da ABM - Internacional e ao 14ํㅡㄹ ENEMET - Encontro Nacional de Estudantes de Engenharia Metalúrgica, de Materiais e de Minas, 21 a 25 de julho de 2014, São Paulo, SP, Brasil.
} 


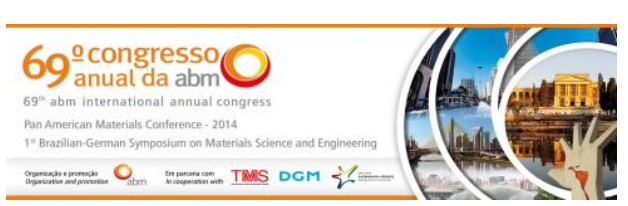

Para realizar o estudo foi utilizado um planejamento fatorial com 4 fatores, cada um deles com dois níveis [11]. Os fatores são as concentrações de $\mathrm{H}_{2} \mathrm{ZrF}_{6}$ e H $\mathrm{H}_{2} \mathrm{TiF}_{6}$, o pH da solução e o tempo de imersão dos corpos de prova em cada solução preparada. Os experimentos foram realizados em duplicata, para obter o erro experimental. $\mathrm{Na}$ Tabela 1 constam os fatores e respectivos níveis.

Tabela 1. Resumo dos fatores e níveis utilizado no planejamento fatorial

\begin{tabular}{lll}
\hline Fatores & Nível baixo & Nível alto \\
\hline Concentração $\mathrm{H}_{2} \mathrm{ZrF}_{6}$ & $2 \mathrm{mmol} / \mathrm{L}$ & $6 \mathrm{mmol} / \mathrm{L}$ \\
\hline Concentração $\mathrm{H}_{2} \mathrm{TiF}_{6}$ & $2 \mathrm{mmol} / \mathrm{L}$ & $6 \mathrm{mmol} / \mathrm{L}$ \\
\hline $\mathrm{pH}$ & 3,5 & 4,5 \\
\hline Tempo de imersão & $90 \mathrm{~s}$ & $210 \mathrm{~s}$ \\
\hline
\end{tabular}

Os corpos de prova foram extraídos de uma chapa de aço galvanizado. Os ácidos $\mathrm{H}_{2} \mathrm{ZrF}_{6}$ e $\mathrm{H}_{2} \mathrm{TiF}_{6}$ foram fornecidos pela Sigma Aldrich. $\mathrm{O} \mathrm{pH}$ das soluções foi ajustado com hidróxido de amônio.

Antes da imersão nas soluções para obtenção do revestimento, os corpos de prova passaram por uma etapa de desengraxe alcalino $(70 \mathrm{~g} / \mathrm{L}$ de Saloclean 667 fornecido por Klintex) entre 65 e $70^{\circ} \mathrm{C}$ e ativação em solução de ácido nítrico $5 \mathrm{~mL} / \mathrm{L}$ por $10 \mathrm{~s}$. Para realização dos ensaios eletroquímicos os corpos de prova foram dispostos como - eletrodo de trabalho em uma célula eletroquímica contendo um eletrodo de referência $\mathrm{Ag} / \mathrm{AgCl}$ e um contra eletrodo de platina imersos em um eletrólito de $\mathrm{NaCl}$ $0,1 \mathrm{~mol} / \mathrm{L}$ e $\mathrm{pH} 5$. As medições foram realizadas utilizando o potenciostato AUTOLAB PGSTAT. A área exposta das amostras à solução salina foi mantida constante em $0,63 \mathrm{~cm} 2$. As medidas de espectroscopia de impedância eletroquímica (EIS) foram realizadas após 1, 2, 6 e $24 \mathrm{~h}$ de contato dos corpos de prova com o eletrólito. A frequência foi variada entre $100 \mathrm{kHz}$ e $10 \mathrm{mHz}$ com amplitude de $10 \mathrm{mV}$ a partir do potencial de circuito aberto medido pelo equipamento. As curvas foram obtidas com o auxílio do software FRA e os valores da resistência total [12] desempenhada por cada amostra foram obtidos com o auxílio do software EIS Spectrum Analyser. A análise dos dados coletados foi realizada utilizando o software Minitab 16.

\section{RESULTADOS E DISCUSSÃO}

O melhor resultado foi obtido para concentração mais baixa, $\mathrm{pH}$ e tempo de imersão de baixos, amostra denominada $A$. A pior condição obtida também foi para a concentração mais baixa, porém com $\mathrm{pH}$ e tempo de imersão altos, amostra denominada B. A Tabela 2 resume as condições testadas que tiveram o melhor e 0 pior desempenho.

Tabela 2. Resumo das condições com melhor e pior desempenhos

\begin{tabular}{lll}
\hline Fatores/Condição testada & Melhor $(\mathrm{A})$ & Pior $(\mathrm{B})$ \\
\hline Concentração $\mathrm{H}_{2} \mathrm{ZrF}_{6}$ & $2 \mathrm{mmol} / \mathrm{L}$ & $2 \mathrm{mmol} / \mathrm{L}$ \\
\hline Concentração $\mathrm{H}_{2} \mathrm{TiF}_{6}$ & $2 \mathrm{mmol} / \mathrm{L}$ & $2 \mathrm{mmol} / \mathrm{L}$ \\
\hline $\mathrm{pH}$ & 3,5 & 4,5 \\
\hline Tempo de imersão & $90 \mathrm{~s}$ & $210 \mathrm{~s}$ \\
\hline
\end{tabular}

Nas Figuras 1 e 2, são apresentados os gráficos Nyquist e Bode para os ensaios de EIS das amostras que tiveram o melhor e o pior desempenho. Observa-se na Figura 1 (a) e (c) comportamento pseudo indutivo nas duas primeiras horas de exposição ao

\footnotetext{
* Contribuição técnica ao 69ำ Congresso Anual da ABM - Internacional e ao 14ํㅡㄹ ENEMET - Encontro Nacional de Estudantes de Engenharia Metalúrgica, de Materiais e de Minas, 21 a 25 de julho de 2014, São Paulo, SP, Brasil.
} 
meio corrosivo [13], que se deve provavelmente às reações de dissolução do $\mathrm{Zn}$ [14].

(a)

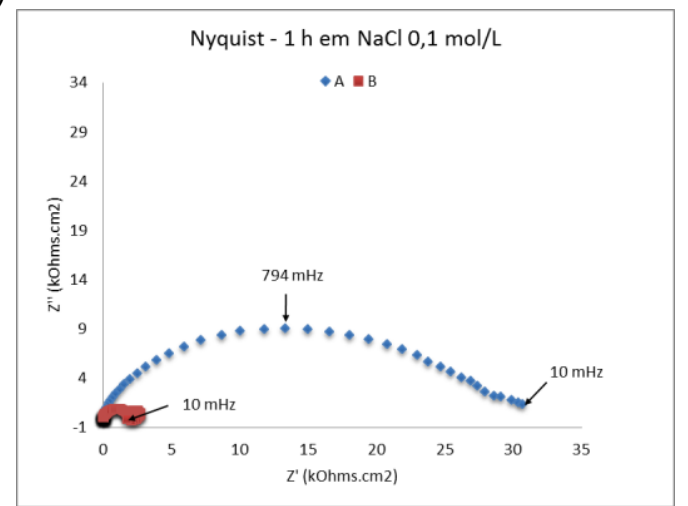

(c)

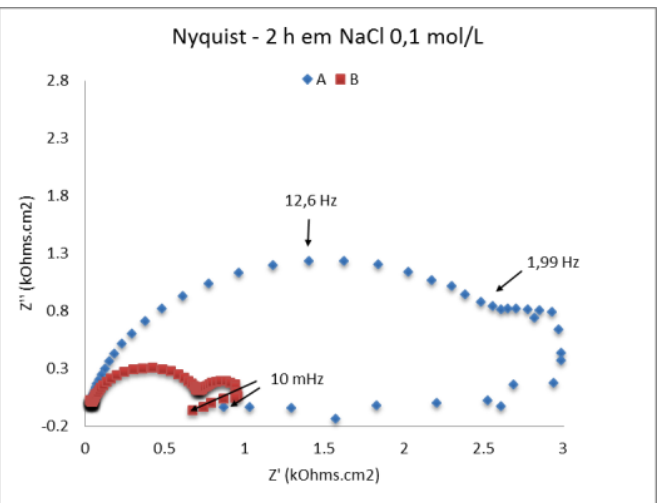

(g)

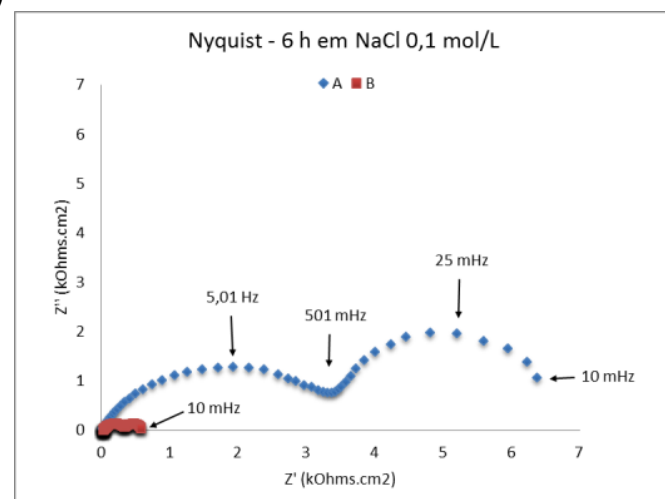

(b)

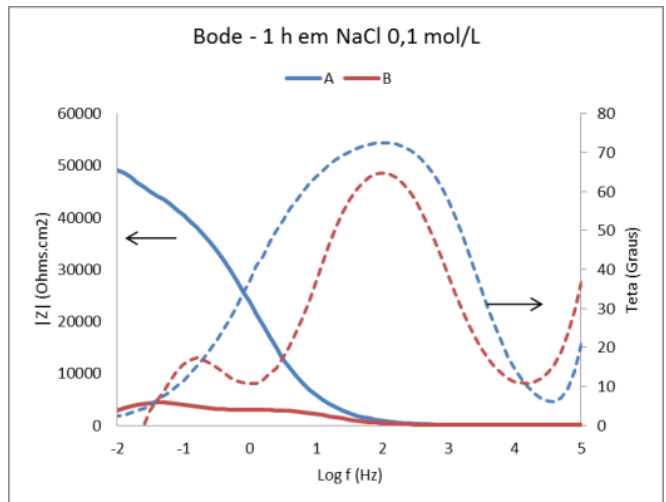

(d)

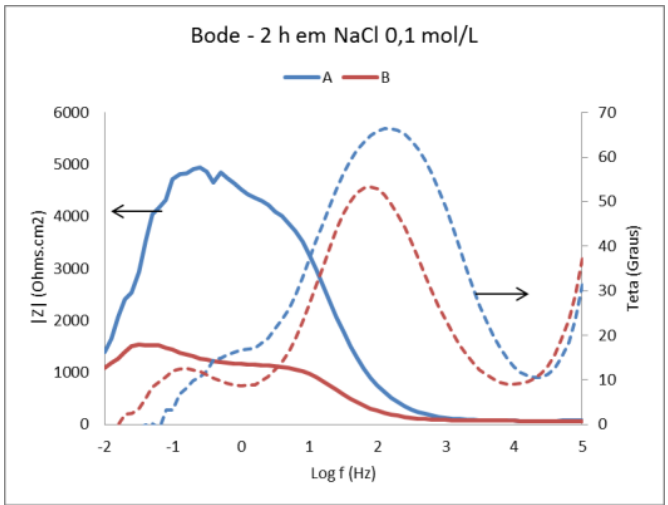

(f)

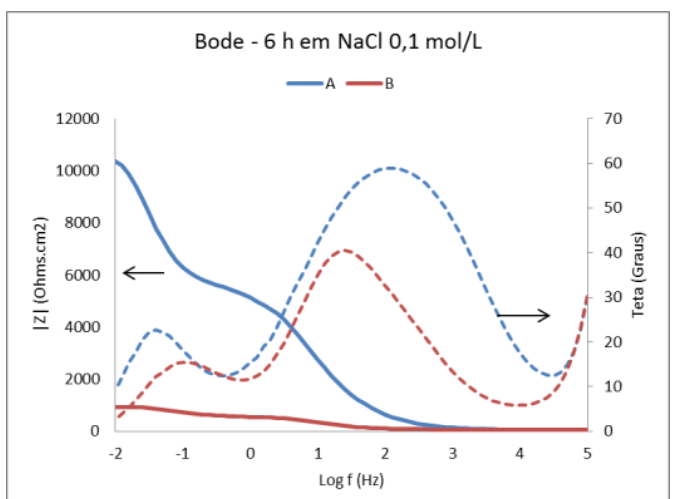

Figura 1. Gráficos de Nyquist e Bode, respectivamente, para as amostras A e B em: $1 \mathrm{~h}(\mathrm{a})$ e (b), $2 \mathrm{~h}$ (c) e (d), 6 h (e) e (f)

O revestimento nanocerâmico apresenta fissuras $[2,15,16]$ que permitem o contato do eletrólito com o substrato e, por consequência, que tais reações ocorram. Após $6 \mathrm{~h}$ de contato com o eletrólito - Figura 1 (e), o comportamento pseudo indutivo desaparece e os sistemas entram em um regime de equilíbrio dinâmico [17]. É notável a diferença entre as resistências apresentadas pelas duas amostras.

Após 24 h em contato com a solução salina - Figura 2 (a) e (b), o desempenho da amostra A cai significativamente, se aproximando ao desempenho da amostra B. Em $48 \mathrm{~h}$ - Figura 2 (c) e (d), o desempenho é praticamente igual.

(a)

* Contribuição técnica ao 69ำ Congresso Anual da ABM - Internacional e ao 14ํㅡㄹ ENEMET - Encontro Nacional de Estudantes de Engenharia Metalúrgica, de Materiais e de Minas, 21 a 25 de julho de 2014, São Paulo, SP, Brasil. 


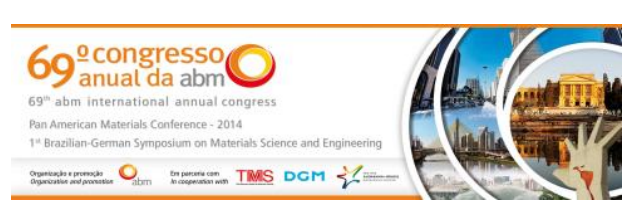

\begin{tabular}{|c|c|c|c|c|c|}
\hline Efeitos Principais & 4 & 15217328 & 3804332 & 3.79 & 0.022 \\
\hline $\mathrm{pH}$ & 1 & 4326211 & 4326211 & 4.32 & 0.053 \\
\hline $\mathrm{Zr}$ & 1 & 4301244 & 4301244 & 4.29 & 0.054 \\
\hline $\mathrm{Ti}$ & 1 & 2672672 & 2672672 & 2.67 & 0.121 \\
\hline Tempo & 1 & 3917201 & 3917201 & 3.91 & 0.065 \\
\hline Interações de segunda ordem & 6 & 40276324 & 6712721 & 6.70 & 0.001 \\
\hline $\mathrm{pH}^{\star} \mathrm{Zr}$ & 1 & 4997541 & 4997541 & 4.99 & 0.039 \\
\hline $\mathrm{pH}^{\star} \mathrm{Ti}$ & 1 & 6703291 & 6703291 & 6.69 & 0.019 \\
\hline $\mathrm{pH}^{\star}$ Tempo & 1 & 2659971 & 2659971 & 2.65 & 0.122 \\
\hline $\mathrm{Zr}^{*} \mathrm{Ti}$ & 1 & 4348300 & 4348300 & 4.34 & 0.053 \\
\hline Zr*Tempo & 1 & 15379058 & 15379058 & 15.34 & 0.001 \\
\hline Ti*Tempo & 1 & 6188162 & 6188162 & 6.17 & 0.024 \\
\hline Interações de terceira ordem & 4 & 41393593 & 10348398 & 10.32 & 0.000 \\
\hline $\mathrm{pH}^{*} \mathrm{Zr}^{*} \mathrm{Ti}$ & 1 & 13679065 & 13679065 & 13.65 & 0.002 \\
\hline $\mathrm{pH}^{\star} \mathrm{Zr}^{*}$ Tempo & 1 & 5990991 & 5990991 & 5.98 & 0.026 \\
\hline $\mathrm{pH}^{*} \mathrm{Ti}^{*}$ Tempo & 1 & 13079055 & 13079055 & 13.05 & 0.002 \\
\hline $\mathrm{Zr}{ }^{*} \mathrm{Ti}^{*} \mathrm{Tempo}$ & 1 & 8644482 & 8644482 & 8.62 & 0.009 \\
\hline Erro Residual & 17 & 17042388 & 1002493 & & \\
\hline Erro Puro & 16 & 9136112 & 571007 & & \\
\hline Total & 31 & 113929633 & & & \\
\hline
\end{tabular}

$\mathrm{O} \mathrm{pH}$ e o tempo possuem pouca interação entre si isoladamente, o que pode ser observado pelo valor $\mathrm{P}$ superior a 0,05 apresentado na Tabela $3\left(\mathrm{pH}^{\star}\right.$ Tempo). Já para as interações entre as concentrações dos ácidos e o tempo de imersão há um comportamento interessante (Valor $\mathrm{P}<0,05$ ): para baixas concentrações um tempo menor de imersão é melhor, já para concentrações mais altas, onde se esperaria uma solução mais agressiva e que necessitaria de menor tempo para se formar, um tempo maior de imersão gera um filme com maior resistência. Ainda não é possível explicar o porque desse resultado.

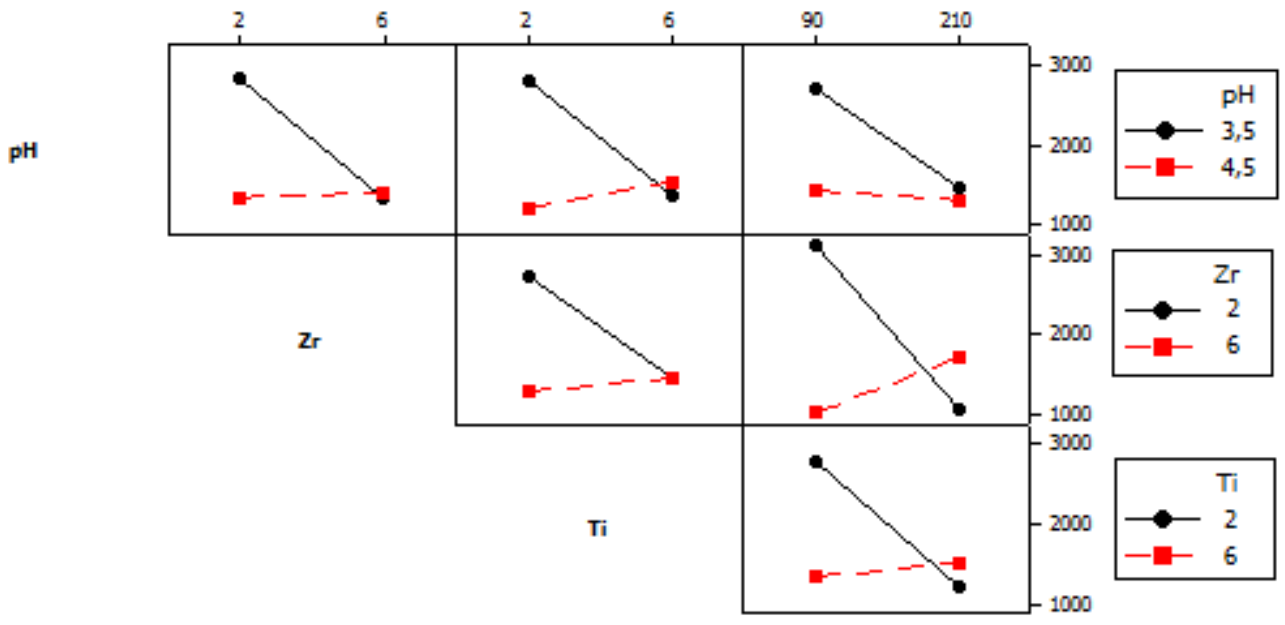

Tempo

Figura 3. Gráficos de interação entre os fatores concentração de $\mathrm{H}_{2} \mathrm{ZrF}_{6}$, concentração de $\mathrm{H}_{2} \mathrm{TiF}_{6}$, pH e tempo de imersão

* Contribuição técnica ao 69ำ Congresso Anual da ABM - Internacional e ao 14ํㅡㄹ ENEMET - Encontro Nacional de Estudantes de Engenharia Metalúrgica, de Materiais e de Minas, 21 a 25 de julho de 2014, São Paulo, SP, Brasil. 
(a)

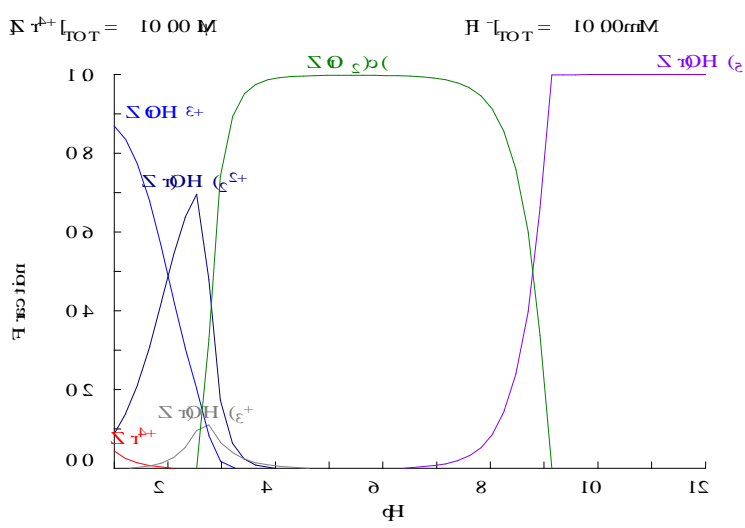

(b)

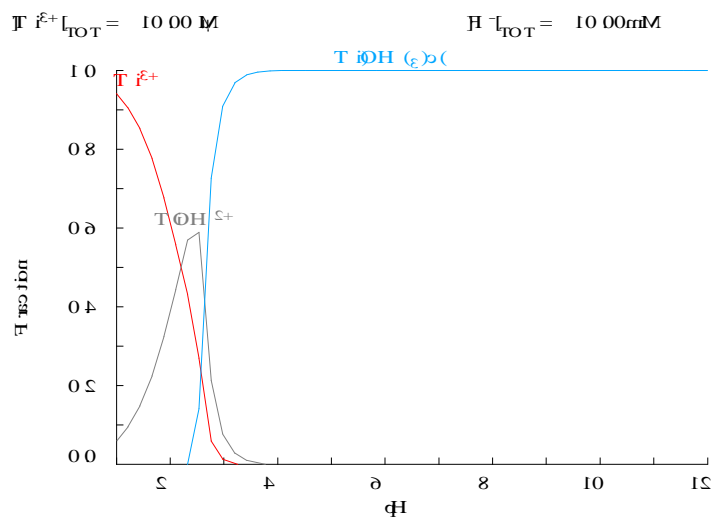

Figura 4. Concentração de espécies químicas em função do $\mathrm{pH}$ para $\mathrm{Zr}$ (a) e Ti (b) gerado pelo software Hydra Medusa

As Figuras 5 e 6 mostram os resultados do experimento fatorial em gráficos de contorno, o que facilita a visualização das considerações feitas anteriormente. $\mathrm{Na}$ Figura 5 (a) temos o gráfico de contorno variando-se o pH e o Tempo e fixando-se as concentrações dos ácidos nos valores mínimos. Na Figura 5 (b) os valores da concentração dos ácidos são fixados nos valores máximos. É interessante observar a formação de uma cela para este último, com os máximos para o maior tempo e menor $\mathrm{pH}$ e na diagonal oposta, menor tempo e maior $\mathrm{pH}$.
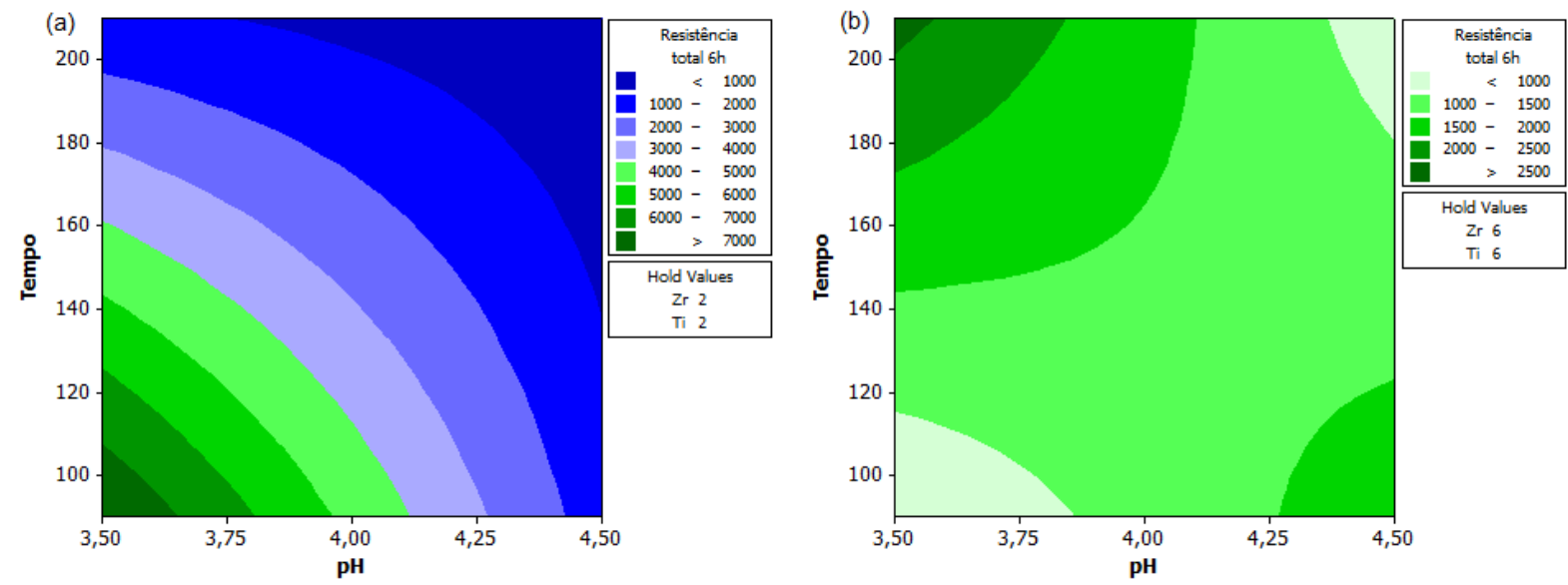

Figura 5. Gráficos de contornos variando as concentrações de e fixando: $2 \mathrm{mmol} / \mathrm{L}$ de $\mathrm{H}_{2} \mathrm{ZrF}_{6}$ e 2 $\mathrm{mmol} / \mathrm{L}$ de $\mathrm{H}_{2} \mathrm{TiF}_{6}(\mathrm{a}) ; 6 \mathrm{mmol} / \mathrm{L}$ de $\mathrm{H}_{2} \mathrm{ZrF}_{6}$ e $6 \mathrm{mmol} / \mathrm{L}$ de $\mathrm{H}_{2} \mathrm{TiF}_{6}$ (b)

Na Figura 6 as concentrações dos ácidos foram variadas, o pH e o tempo foram fixados nos valores mínimos em (a) e nos valores máximos em (b). A mesma mudança de comportamento para uma cela é observada em (b).

\footnotetext{
* Contribuição técnica ao 69ำ Congresso Anual da ABM - Internacional e ao 14ํㅡㄹ ENEMET - Encontro Nacional de Estudantes de Engenharia Metalúrgica, de Materiais e de Minas, 21 a 25 de julho de 2014, São Paulo, SP, Brasil.
} 

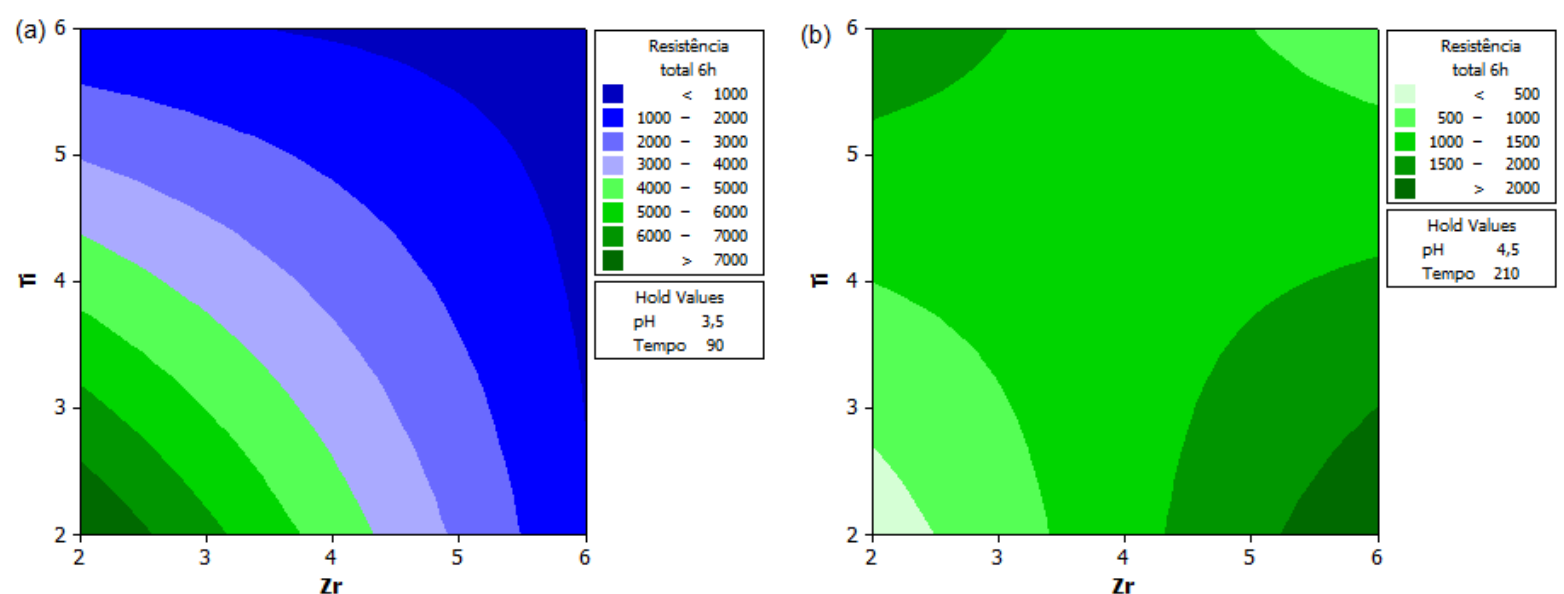

Figura 6. Gráficos de contornos variando as concentrações de H2ZrF6 e H2TiF6 e fixando: pH 3,5 e Tempo $90 \mathrm{~s}(\mathrm{a}) ; \mathrm{pH} 4,5$ e Tempo 210 (b)

\section{CONCLUSÃO}

A amostra que obteve o melhor desempenho frente à corrosão foi a amostra feita com a solução com a menor concentração dos ácidos hexafluorotitânio e hexafluorozircônio, pH 3,5 e tempo de imersão de $90 \mathrm{~s}$. As interações de terceira ordem entre os fatores são significativas, assim para o controle de processo todas as variáveis estudadas precisam ser corretamente ajustadas para um desempenho ótimo do revestimento.

\section{Agradecimentos}

Agradeço à equipe do LACOR pela estrutura e agregação de conhecimento nas discussões sobre o tema, ao PPGE3M, à UFRGS e ao CAPES, financiador da bolsa de mestrado acadêmico.

\section{REFERÊNCIAS}

1 Fedrizzi L, Deflorian F, Bonora PL, Pasini E. EIS study of environmentally friendly coil coating performances. Progress in Organic Coatings. 1996, 29: 89-96.

2 Mohammadloo HE, Sarabi AA, Alvani AAS, Sameie H, Salimi R. Nano-ceramic hexafluorozirconic acid based conversion thin film: Surface characterization and electrochemical study. Surface and Coatings Technology, 2012; 206: 4132-4139.

3 Andreatta F, Turco A, Graeve I, Terryn H, Wit JHW, Fedrizzi L. SKPFM and SEM study of the deposition mechanism of $\mathrm{Zr} / \mathrm{Ti}$ based pre-treatment on AA6016 aluminum alloy. Surface \& Coatings Technology. 2007, 201:7668-7685.

4 Fedrizzi L, Deflorian F, Bonora PL. Corrosion behaviour of fluotitanate pretreated and painted aluminium sheets. Electrochimica Acta. 1997,42: 969-978.

5 Nordlien JH, Walmsleya JC, Østerbergb H, Nisancioglub K. Formation of a zirconiumtitanium based conversion layer on AA 6060 aluminium. Surface and Coatings Technology. 2002;153:72-78.

6 Hamdy ASE, Farahat M. Chrome-free zirconia-based protective coatings for magnesium alloys. Surface \& Coatings Technology. 2010;204:2834-2840.

7 Stromberg C, Thissen P, Klueppel I, Fink N, Grundemeier G. Synthesis and characterisation of surface gradient thin conversion films on zinc coated steel. Electrochimica Acta, 2006; 52: 804 - 815.

\footnotetext{
* Contribuição técnica ao 69ำ Congresso Anual da ABM - Internacional e ao 14ํㅡㄹ ENEMET - Encontro Nacional de Estudantes de Engenharia Metalúrgica, de Materiais e de Minas, 21 a 25 de julho de 2014, São Paulo, SP, Brasil.
} 


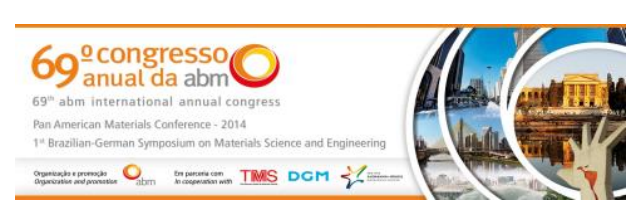

8 Deck PD, Moon M, Sujdak RJ. Investigation of fluoacid based conversion coatings on aluminum. Progress in Organic Coatings, 1998; 34: 39-48.

9 Lunder O, Simensen C, Yu Y, Nisancioglu K. Formation and characterisation of $\mathrm{Ti}-\mathrm{Zr}$ based conversion layers on AA6060 aluminium. Surface and Coatings Technology, 2004; 184: 278-290.

10 Verdier S, Laakb N, Dalarda F, Metsonc J, Delalande S. An electrochemical and SEM study of the mechanism of formation,morphology and composition of titanium or zirconium fluoride-based coatings. Surface \& Coatings Technology, 2006; 200: 29552964.

11 Montgomery DC. Design and analysis of experiments, John Wiley \& Son, 2001.

12 Wolynec S. Técnicas eletroquímicas em corrosão. São Paulo: Editora da Universidade de São Paulo, 2003.

13 Epelboin I, Keddam M, Takenouti $H$. Use of impedance measurements for the determination of the instant rate of metal corrosion. Journal of Applied Electrochemistry, 1972; 2: 71-79.

14 Giménez-Romero D, García-Jareño JJ, Vicente F. EQCM and EIS studies of $\mathrm{Zn}_{\mathrm{aq}}{ }^{2+}+2 \mathrm{e}^{-}$ $\leftrightarrows \mathrm{Zn}^{0}$ electrochemical reaction in moderated acid medium. Journal of Electroanalytical Chemistry. 2003, 558: 25-33.

15 Pareja RR, Ibáñez RL, Martín F, Ramos-Barrado JR, Leinen D. Corrosion behaviour of zirconia barrier coatings on galvanized steel. Surface and Coatings Technology, 2006; 200; 6606- 6610.

16 Tsai YT, Hou KH, Bai CY, Lee JL, Ger MD. The influence on immersion time of titanium conversion coatings on electrogalvanized steel. Thin Solid Films, 2010; 518: 7541-7544.

17 Paussa L, Andreatta F, Aldighieri P, Fedrizzi L. Critical apsects in the electrochemical study of unstable coated metallic substrates. Progress in Organic Coatings. 2010; 69:225-234.

* Contribuição técnica ao 69ำ Congresso Anual da ABM - Internacional e ao 14ํㅡㄹ ENEMET - Encontro Nacional de Estudantes de Engenharia Metalúrgica, de Materiais e de Minas, 21 a 25 de julho de 2014, São Paulo, SP, Brasil. 